\title{
COMMUNICATION
}

\section{Innovation dans la communication et la gestion du stress en chirurgie orale : méthode d'analyse ProcessCom ${ }^{\circledR}$}

\author{
S. Maizeray (Brest), H. Herry (Brest), G. Valette (Brest), S. Boisramé (Brest)
}

Pas facile de savoir communiquer efficacement en tant que chirurgien oral ! C'est probablement la raison pour laquelle nous parlons d'art pour un acte aussi banal que celui de communiquer avec son patient. L'art de communiquer, c'est l'art de " tester »son interlocuteur pour se positionner rapidement dans sa personnalité et mettre en place le bon canal de communication. Les situations de stress s'imposent quotidiennement pour le patient et pour le chirurgien. Et la résolution de ces situations dépend de la qualité de communication car c'est la qualité de l'entente interpersonnelle qui va influencer celle du traitement, de la santé buccale du patient et du bien être du praticien. De nombreuses thérapeutiques sont actuellement utilisées pour faciliter la gestion du stress : la prémédication sédative (antihistaminiques, benzodiazépines, ), la sédation consciente, la psychothérapie (thérapies cognitives comportementales), l'hypnose, la musicothérapie Créée au début des années 1980 par T. Kahler, la Process Communication (PC) est un outil de compréhension du fonctionnement des différentes parties de la personnalité présentes à des degrés variables chez une personne. Cette méthode d'analyse a démontré un intérêt majeur en tant qu'outil positif de communication lors de séminaires de formation et pour les étudiants et enseignants (Hranicky, 2015) (Drouin 2015) (Collignon, 2013). La manière de dire les choses dans la PC a plus d'importance que le contenu du message. Dans une situation dite "stressante ", le problème vient rarement de ce qui est dit mais surtout de la façon dont cela a été dit. La PC apporte une carte rapidement lisible des caractéristiques d'un type de personnalité : ses points forts, ses motivations, ses modes de communication, les environnements dans lesquels il sera à l'aise ou pas, les types de personnalité avec lesquels il interagira facilement ou difficilement. Il existe donc des stratégies individuelles face à chaque personnalité pour éviter les situations de «mécommunication» et pour retrouver la disponibilité intellectuelle et émotionnelle. Le premier intérêt de ce modèle est de s'occuper de soi ; c'est à dire identifier nos points forts et nos motivations qui permettent d'accroître notre flexibilité et efficacité à communiquer, agir sur nos comportements de stress et utiliser au mieux nos talents personnels. Le second intérêt est de s'occuper de l'autre; c'est-à-dire savoir de quoi il a besoin dans sa relation. Ce modèle de communication présente un grand intérêt dans la prise en charge du patient stressé et transmetteur de stress en chirurgie orale. Il nous amène à réaliser que les difficultés de la communication ne sont pas à imputer au type de personnalité de l'autre mais à la relation dysfonctionnelle existante entre deux types de personnalité. Ce changement de croyance ou d'attitude mentale, vis-à-vis de l'autre constitue un pré-requis indispensable à l'amélioration de la flexibilité relationnelle et du professionnalisme du chirurgien oral. De futures recherches en santé seront à mener afin de diversifier les applications de cette méthode, notamment en chirurgie orale. 